\title{
Free Vibration for an Electromagnetic Harmonic Movable Tooth Drive System
}

\author{
Yongli Liang and Lizhong $\mathrm{Xu}^{*}$
}

Mechanical Engineering Institute, Yanshan University, Qinhuangdao, 066004, China

\begin{abstract}
The authors proposed an electromagnetic harmonic movable tooth drive system which is an electromechanical coupled system where the coupled dynamics controls its overall operating behavior. However, the coupled dynamics of such a system was not found in the literature. In this paper, an electromechanical coupled dynamics equation of the flexible ring subjected to electromagnetic force is presented. Using the equation, the natural frequencies and vibration modes of the flexible ring are investigated. The results show that the natural frequency of the drive system is affected by mechanical and electrical parameters among which, the coil current, the average static displacement of the flexible ring and the air gap have more obvious influence on the natural frequencies. The results are useful for the design and further study of the dynamics performance for the drive system.
\end{abstract}

Keywords: Electromagnetic drive, Free vibration, Movable tooth drive, Harmonic drive, Natural frequency, Vibrating modes.

\section{INTRODUCTION}

The electromagnetic harmonic drive was proposed by D. F. Herdeg [1]. It is suitable for the technical fields such as aviation and space flight, etc. Besides the above-mentioned fields that require compactness, the drive can be used in fields such as robots, etc., that require accurate control. Some countries have been developing the harmonic drives for years [2-4].

For the electromagnetic harmonic drives, Janes proposed a type of the electromagnetic force change to avoid eddy current through the metal flexible ring [5]. Software was used to realize the controlling of high performance servo driving system for magnetic type harmonic gear drive [6]. Rens investigated a novel magnetic harmonic gear drive which has high reliability, and inherent overload protection while having a high efficiency [7]. Another new electromagnetic harmonic drive was investigated in which oscillating teeth transmission principle was used [8]. Liu established an analytical model of the eccentric magnetic harmonic gear based on the boundary perturbation method and calculated the eccentric air-gap magnetic field [9]. Uchimura proposed a control method of the harmonic magnetic gear to attenuate adverse effects due to cogging torque on the vibratory system [10].

Compared with the mechanical harmonic drive, electromagnetic harmonic drive has a small size. However, the mechanical harmonic drive has a large output torque. For the mechanical harmonic drives, Dhaouadi et al proposed a mathematical model and its parameter identification scheme for harmonic drive gears with compliance and hysteresis [11]. Dong et al completed the dynamic simulation of harmonic

*Address correspondence to this author at the Mechanical Engineering Institute, Yanshan University, Hebei Street, Qinhuangdao, Postcard: 066004, China; Tel: 0335-8057031; E-mail: xlz@ysu.edu.cn gear drives [12]. Zhu et al proposed a new type of gear pump based on the principle of harmonic gear drive [13].

In a word, a number of studies about the harmonic drives were done. However, for the mechanical harmonic drive, the teeth had to be produced on the flexible ring which is a difficult task. Hence, the authors proposed an electromagnetic harmonic movable tooth drive system in which the teeth are removed from the flexible ring.

The configuration of the proposed electromagnetic harmonic movable tooth drive system is shown in Fig. (1), which consists of three main parts: electromagnetic coils, harmonic movable tooth drive without wave generator, and flexible ring between the coils and the drive.

The flexible ring is made with ferromagnetic material. The coils and the rigid ring are fixed on the housing. The coils are energized sequentially by voltages, and the resulting rotational electromagnetic field causes periodic elastic deformation of the flexible ring. This deformation is accompanied by periodic contact between the flexible ring and the movable teeth. It causes the normal pressure and the relative motion between the movable teeth and rigid ring. As the rigid ring is fixed, the meshing force drives the rotor to rotate. One circle of the electromagnetic field corresponds to two tooth distances of the movable tooth motion, and then a reduction ratio occurs and a large output torque can be obtained.

Compared with other harmonic drives, the drive system has the main advantages as below: (1) rolling contact, transmitting load by meshing, and reduction; (2) high operating efficiency; and (3) small size.

The undesirable dynamic behavior of the drive system will result in noise and unacceptable performance characteristics. The prediction of the natural frequencies and vibration modes is required in the design stage for a drive system. However, 
the free vibration of electromagnetic harmonic movable tooth drive system is yet to be developed.
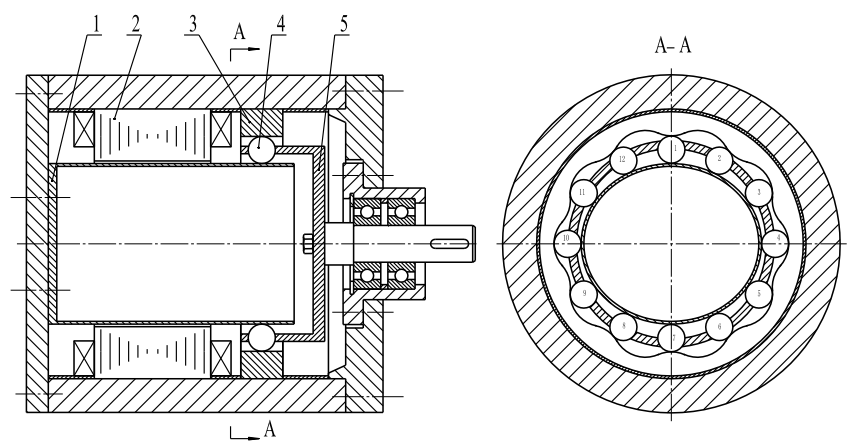

Fig. (1). An electromagnetic harmonic movable tooth drive system 1. flexible ring; 2 . coils; 3 . rigid gear; 4 . movable tooth; 5 . rotor.

In this paper, an electromagnetic harmonic movable tooth drive system is proposed, an electromechanical coupled force is analyzed, and a dynamics equation of the flexible ring subjected to electromagnetic force is presented. From the equation, the equations of the natural frequencies and vibration modes of the flexible ring are developed. Using these equations, the natural frequencies and vibration modes of the drive system are investigated. A number of the useful results are obtained. The results show that the natural frequency of the electromagnetic harmonic movable tooth drive system is affected by mechanical and electrical parameters among which, the coil current, the average static displacement of the flexible ring, and the air gap have more obvious influence on the natural frequencies. The results are useful for the design and further study of the dynamics performance for the drive system.

\section{ELECTROMECHANICAL COUPLED FORCE}

The flexible ring subject to the electromagnetic force is shown in Fig. (2). The radial displacement of the flexible ring under one force $F$ is

$$
\begin{aligned}
& w=\frac{F r^{3}}{K l} \sum_{n=2,4,6 \ldots}^{\infty}\left\{\frac{1}{\left(\begin{array}{ll}
n^{2} & 1
\end{array}\right)^{2}}\right. \\
& \left.+\frac{n^{2} c x}{\left(\begin{array}{ll}
n^{2} & 1
\end{array}\right)^{2}\left[\frac{1}{3} n^{2} l^{2}+2\left(\begin{array}{ll}
1 & ) r^{2}
\end{array}\right]\right.}\right\} \cos n
\end{aligned}
$$

where $w$ is the radial displacement of the flexible ring; $x, y$ and $\theta$ are the coordinates on the flexible ring; $l$ is the length of the flexible ring; $r$ denotes the radius of the flexible ring; $K$ is the bending stiffness of the flexible ring, $K=\frac{E t^{3}}{12(1)}$. Here, $E$ is the modulus of elasticity of the ring material, $\gamma$ is the Poisson's ratio. $t$ is the thickness of the flexible ring; $c$ is the position of the force; $n$ is the positive integer; $x$ is the position of the displacement.

For the distributed electromagnetic force, the radial displacement of the flexible ring can be given as

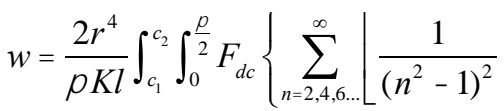

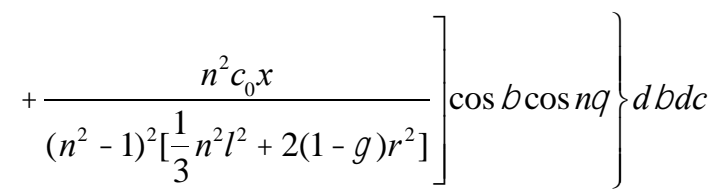

where $c_{1}$ and $c_{2}$ are the initial points and the end point of the electromagnetic force in the $x$ direction, respectively; $c_{0}=\left(c_{1}+c_{2}\right) / 2 ; \beta$ is the angle position of the force point; $F_{\mathrm{dc}}$ is the distributed electromagnetic force as the function of the position coordinates $\beta$ and $c$.

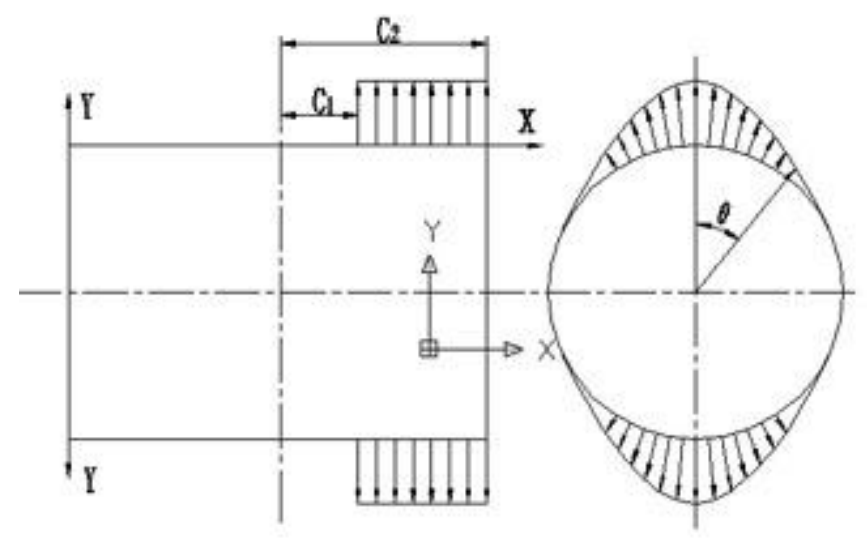

Fig. (2). The flexible ring subject to the electromagnetic force.

At $c=c_{0}$, the displacement of the flexible ring is the maximum at $\theta=0$. From Eq. (2), it can be obtained

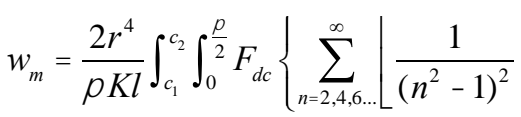

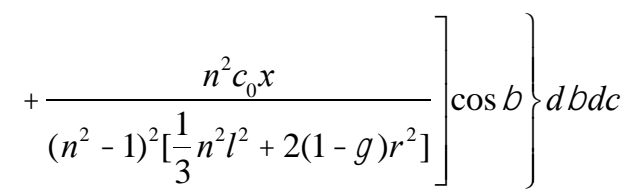

The magnetic induction intensity caused by the air gap magnetic potential as the function of the position coordinate $\theta$ can be given as

$$
B=\frac{B_{0}}{w_{m} \cos 2}
$$

where $B_{0}=\frac{{ }_{0} F_{1}}{{ }_{0}}=1.35 \frac{{ }_{0} N I}{{ }_{0} p} ; \mu_{0}$ is the magnetic conductivity of free space, $\mu_{0}=4 \pi \times 10^{-7} \mathrm{~W} / \mathrm{m}$.A; $N$ is the coil number; $I$ is the coil current; $\delta_{0}$ is the initial gas gap; $p$ is the pole pair number.

From Eq. (4), one can obtain the radial electromagnetic force per unit area on the flexible ring

$$
F_{d c}=\frac{B^{2}}{2_{0}}=\frac{B_{0}^{2}}{2_{0}\left(w_{0} w_{m} \cos 2\right)^{2}} S_{i}
$$

Letting $K_{t}=\frac{w_{m}}{}$, and substituting it and (5) into (3), yields 


$$
\begin{aligned}
w_{\mathrm{m}}= & \frac{2 r^{4}}{K l} \cdot \frac{B_{0}{ }^{2}\left(c_{2} c_{1}\right)}{2_{0}\left(1 \quad K_{t}\right)^{2}} \\
& \cdot \sum_{n=2,4,6 \ldots}^{\infty}\left[\frac{1}{\left(\begin{array}{ll}
n^{2} & 1
\end{array}\right)^{2}}+\frac{n^{2} c_{0}{ }^{2}}{\left(\begin{array}{ll}
n^{2} & 1
\end{array}\right)^{2}\left[\begin{array}{ll}
\frac{1}{3} n^{2} l^{2}+2(1 & ) r^{2}
\end{array}\right]}\right]
\end{aligned}
$$

Letting

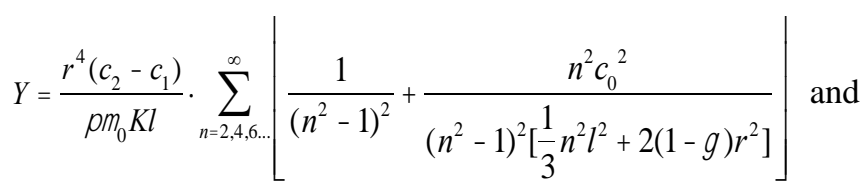
$w_{m}=K_{w} \delta_{0} \quad\left(0 \leq K_{w} \leq 1\right)$, ones can obtain from Eq. (6)

$I=\sqrt{\frac{{ }_{0}^{3} p^{2} K_{w}\left(1 \quad K_{w}\right)^{2}}{1.8225 N^{2} Y}}$

Eq. (7) gives the relationship between the coil current and the displacement of the flexible ring.

\section{FREE VIBRATION}

The dynamic equation of the flexible ring subjected to electromagnetic force is

$$
\frac{\partial^{4} w}{\partial^{4}}+2 \frac{\partial^{2} w}{\partial^{2}}+w=\frac{R_{m}^{4}}{E I_{x}} q_{r} \frac{R_{m}{ }^{4} A}{E I_{x}} \frac{\partial^{2} w}{\partial t^{2}}
$$

where $\Delta w$ is the dynamic displacement of the flexible ring, $R_{\mathrm{m}}$ is the average radius of the flexible ring, $I_{x}$ is section modular of the ring, $\rho$ is material density of the ring, $A$ is its cross section area, $t$ is the time, $q_{r}$ is the dynamic electromagnetic force per unit arc length on the flexible ring.

The dynamic electromagnetic force can be calculated as

$$
q_{r}=\frac{d q_{r}}{d w} \quad w=\frac{d F_{d c}}{d w} \quad w=\frac{B_{0}^{2}{ }^{2}}{{ }_{0}\left(\begin{array}{cc}
0 & w
\end{array}\right)^{3}} w
$$

Substituting Eq. (9) into Eq. (8), yields

$$
\frac{\partial^{4} w}{\partial^{4}}+2 \frac{\partial^{2} w}{\partial^{2}}+w=\frac{R_{m}^{4} B_{0}^{2}{ }_{0}^{2}}{E I_{x_{0}}\left({ }_{0} w\right)^{3}} w \frac{R_{m}{ }^{4} A}{E I_{x}} \frac{\partial^{2} w}{\partial t^{2}}
$$

Eq. (10) is just the electromechanical coupled dynamics equation of the flexible ring subjected to electromagnetic load.

Let $w=() q(t)$, substituting it into (10), we obtain

$$
\frac{\ddot{q}(t)}{q(t)}=\frac{{ }_{1}^{(4)}()+2_{1}{ }^{\prime \prime}()+P_{1}()}{\frac{A R_{m}{ }^{4}}{E I_{x}}()}
$$

where $P=1 \frac{R_{m}^{4} B_{0}^{2}{ }^{2}}{E I_{x} \quad{ }_{0}\left(\begin{array}{ll} & w\end{array}\right)^{3}}$

Let Eq. (11) equal constant $\quad{ }^{2}$, thus

$$
\begin{aligned}
& \ddot{q}(t)+{ }^{2} q(t)=0 \\
& { }_{1}^{(4)}()+2_{1}^{\prime \prime}()+Q_{1}()=0 \\
& \text { where } Q=P \frac{A R_{m}^{4}}{E I_{x}}{ }^{2}
\end{aligned}
$$

Eq. (12) is dynamic equation of linear single degree of freedom system. Its general solution is

$$
q(t)=\sin (t+p)
$$

The mode function of the flexible ring radial vibration is considered as

()$=e$

Substituting Eq. (15) into (13), then

$$
{ }^{4}+2^{2}+Q=0
$$

Solutions of Eq. (16) are $\pm \sqrt{1+\sqrt{1 Q}}$ and $\pm i \sqrt{1+\sqrt{1 Q}}$. Let $m_{1}=\sqrt{1+\sqrt{1 Q}} \quad$ and $m_{2}=\sqrt{1+\sqrt{1 Q}}$, then general solution of Eq. (13) is

$$
{ }_{1}(\quad)=A_{1} \cos m_{1}+A_{2} \sin m_{1}+A_{3} c h m_{2}+A_{4} \operatorname{sh} m_{2}
$$

Owing to flexible ring and its load symmetry, it is known that at $\theta=0$ and $\theta=\frac{\pi}{2}$, rotational angle of normal, and shear force. Thus $\varphi_{1}^{\prime}(\theta)_{\theta=0}=0, \varphi_{2}^{\prime}(\theta)_{\theta=\frac{\pi}{2}}=0, \varphi_{1}^{(3)}(\theta)_{\theta=0}=0$, and $\varphi_{2}^{(3)}(\theta)_{\theta=\frac{\pi}{2}}=0$

Substituting Eq. (17) into the boundary conditions, then

$C_{1} X_{1}=D_{1}$

where

$$
\begin{aligned}
& C_{1}=\left|\begin{array}{cccc}
0 & m_{1} & 0 & m_{2} \\
-m_{1} \sin \left(\frac{\pi}{2} m_{1}\right) & m_{1} \cos \left(\frac{\pi}{2} m_{1}\right) & m_{2} \operatorname{sh}\left(\frac{\pi}{2} m_{2}\right) & m_{2} \operatorname{ch}\left(\frac{\pi}{2} m_{2}\right) \\
0 & -m_{1}^{3} & 0 & m_{2}^{3} \\
m_{1}^{3} \sin \left(\frac{\pi}{2} m_{1}\right) & -m_{1}^{3} \cos \left(\frac{\pi}{2} m_{1}\right) & m_{2}{ }^{3} \operatorname{sh}\left(\frac{\pi}{2} m_{2}\right) & m_{2}{ }^{3} \operatorname{ch}\left(\frac{\pi}{2} m_{2}\right)
\end{array}\right| \\
& X_{1}=\left[\begin{array}{llll}
A_{1} & A_{2} & A_{3} & A_{4}
\end{array}\right]^{T} \quad D_{1}=\left[\begin{array}{llll}
0 & 0 & 0 & 0
\end{array}\right]^{T}
\end{aligned}
$$

The condition that non-zero coefficients $A_{j}(j=1,2,3,4)$ exist is

$$
\left|\begin{array}{cccc}
0 & m_{1} & 0 & m_{2} \\
m_{1} \sin \left(\frac{-}{2} m_{1}\right) & m_{1} \cos \left(\frac{\left.-m_{1}\right)}{2}\right. & m_{2} \operatorname{sh}\left(\frac{\left.-m_{2}\right)}{2}\right. & m_{2} \operatorname{ch}\left(\frac{-}{2} m_{2}\right) \\
0 & m_{1}{ }^{3} & 0 & m_{2}{ }^{3} \\
m_{1}{ }^{3} \sin \left(\frac{-}{2} m_{1}\right) & m_{1}{ }^{3} \cos \left(\frac{-}{2} m_{1}\right) & m_{2}{ }^{3} \operatorname{sh}\left(\frac{-}{2} m_{2}\right) & m_{2}{ }^{3} \operatorname{ch}\left(\frac{-}{2} m_{2}\right)
\end{array}\right|=0
$$

From Eq. (19), we can obtain 


$$
\left(m_{1} m_{2}^{3}+m_{1}^{3} m_{2}\right)^{2} \sin \left(\frac{-}{2} m_{1}\right) \operatorname{sh}\left(\frac{-}{2} m_{2}\right)=0
$$

From Eq. (20), the natural frequencies of the flexible ring vibrations can be calculated. Then, substituting these natural frequencies into Eq. (17), let $A_{1}=1$, other constants $A_{j}$ can be obtained

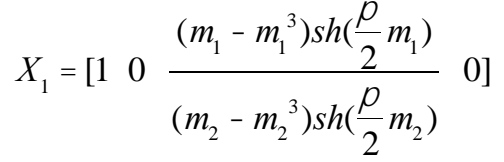

Substituting Eq. (21) into (17), the mode function can be given by

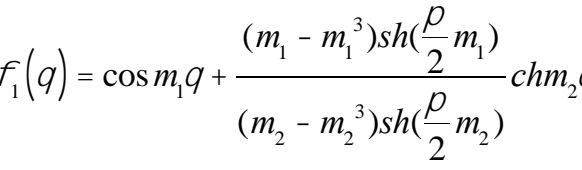

\section{RESULTS}

Consider an electromagnetic harmonic movable tooth drive system defined by the data given in Table 1. The material used for the flexible ring is steel. It is subjected to an electromagnetic field. By Eq. (7), the variation of the coil current with respect to the flexible ring displacement coefficient $k_{\mathrm{W}}$ is obtained as shown in Fig. (3). From Fig. (3), it is seen that there is an extreme current point, after which the flexible ring buckles. So, the operating current of the drive system should not be taken more than the extreme current in order to operate normally of the drive system.

Table 1. Parameters of drive system.

\begin{tabular}{|l|c|}
\hline outer radius of flexible ring $R(\mathrm{~mm})$ & 39 \\
\hline thickness of flexible ring $\tau(\mathrm{mm})$ & 0.2 \\
\hline length of flexible ring $l(\mathrm{~mm})$ & 140 \\
\hline coil number $n$ & 81 \\
\hline Pole pair number $p$ & 3 \\
\hline inter radius of rotor $R_{\mathrm{n}}(\mathrm{mm})$ & 40 \\
\hline
\end{tabular}

Eq. (20) is utilized for the analysis of the natural frequencies of the electromagnetic harmonic movable tooth drive system. Then, substituting these natural frequencies into Eq. (22), the vibration modes of the flexible ring can be obtained. The parameters of the numerical example are shown in Table 1. Table 2 lists the first four natural frequencies of the flexible ring. The changes of the natural frequencies along with the main parameters are shown in Fig. (4). The vibration modes of the flexible ring are shown in Fig. (5). From Fig. (4), the following observations are worth noting:

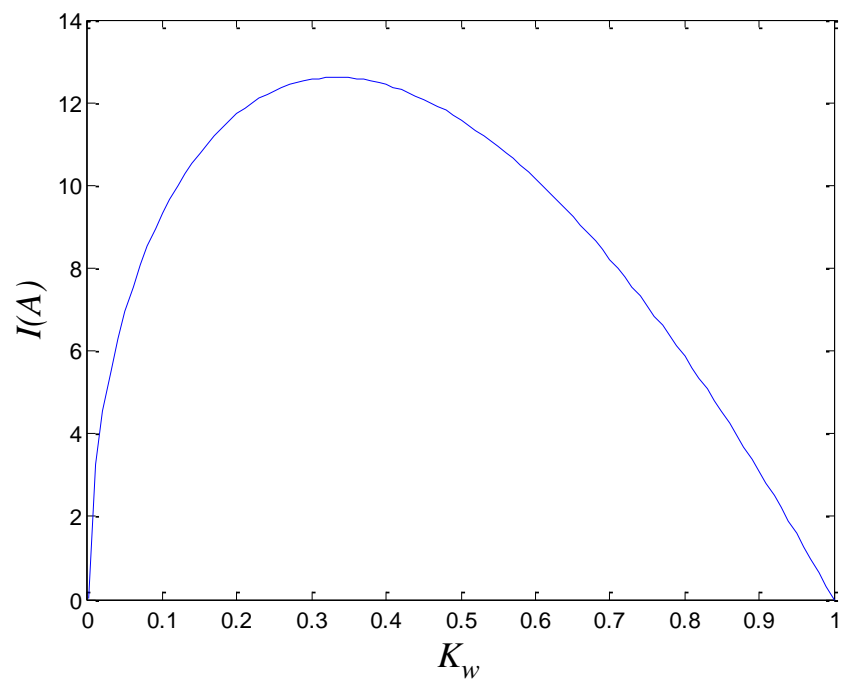

Fig. (3). Changes of current along with displacement.

(1) As the coil current $I$ increases, the natural frequencies of the drive system decrease gradually. It is because the electromagnetic force can cause decrease of the flexible ring. As the order number of the modes grows, the effects of the coil current on the natural frequencies become small. As the coil current grows, the effects of the coil current on the natural frequencies become large. So, the effects should be considered for a large coil current and the low order modes.

(2) As the thickness $\tau$ of the flexible ring increases, the natural frequency of the drive system increases gradually. The natural frequency increases rapider with increasing thickness $\tau$ of the flexible ring when the thickness $\tau$ of the flexible ring is relatively small. As the order number of the modes grows, the effects of the thickness $\tau$ of the flexible ring on the natural frequencies become small as well. So, the effects of the thickness $\tau$ of the flexible ring on the natural frequencies are significant for a small 1 thickness $\tau$ and the low order modes.

(3) As the clearance $\delta_{0}$ between the flexible ring and coils increases, the natural frequency of the drive system increases gradually. It is because a large clearance $\delta_{0}$ between the flexible ring and coils can cause decrease of the electromagnetic force for a given coil current. For the low order modes and a small clearance $\delta_{0}$, the effects of the clearance $\delta_{0}$ of the flexible ring on the natural frequencies are more obvious.

(4) As flexible ring radius $R_{\mathrm{m}}$ increases, the natural frequency of the drive system decreases. The smaller the radius $R_{\mathrm{m}}$ is, the more rapidly the natural frequency decreases. As the order number of the modes grows, the effects of the flexible ring radius $R_{\mathrm{m}}$ on the natural frequencies become large. So, that the effects should be considered for a small flexible ring radius $R_{\mathrm{m}}$ and the high order modes. 
(a) $I$ changes
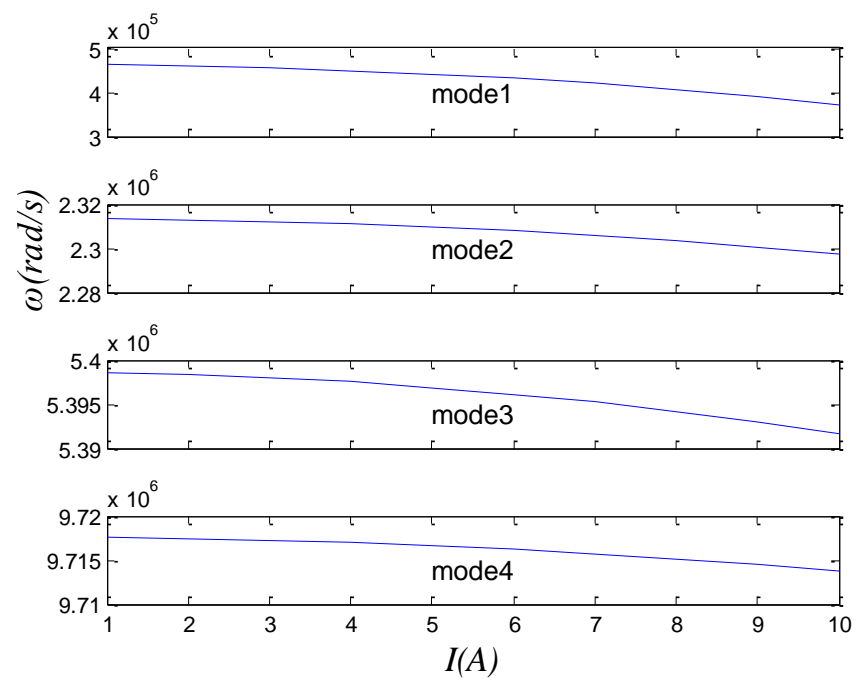

(b) $\tau$ changes

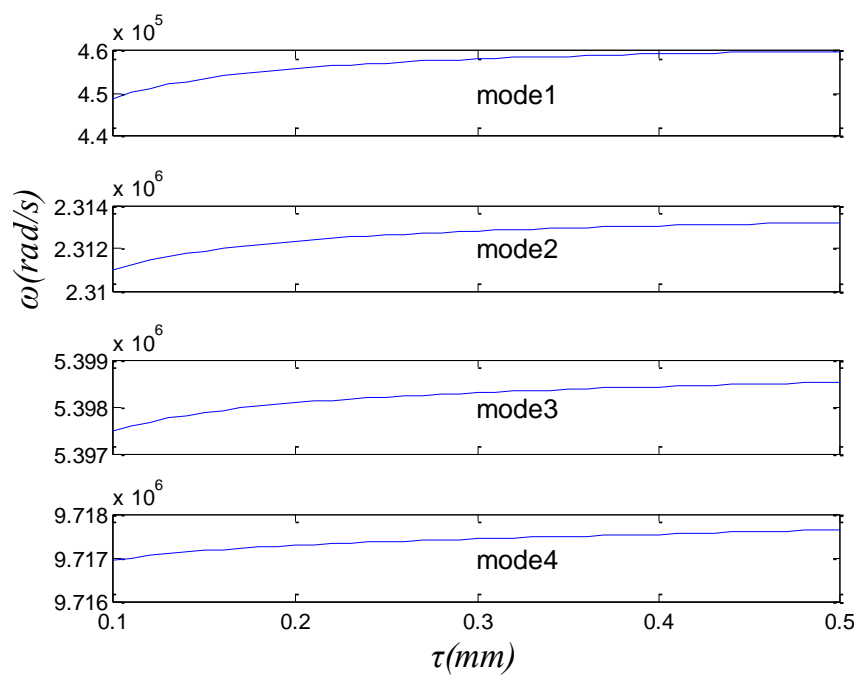

(c) $\delta_{0}$ changes

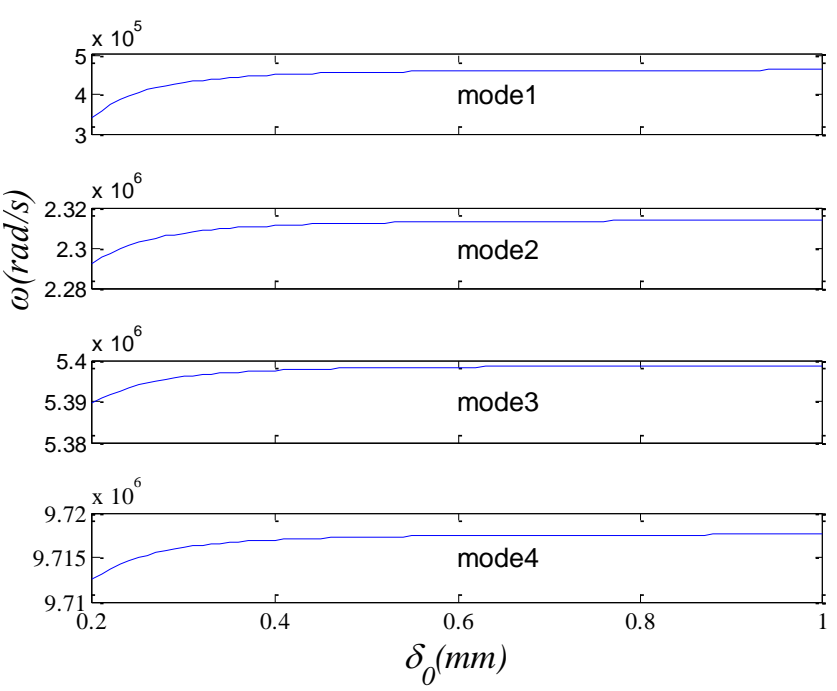

(Fig. 4) contd.....

(d) $r$ changes

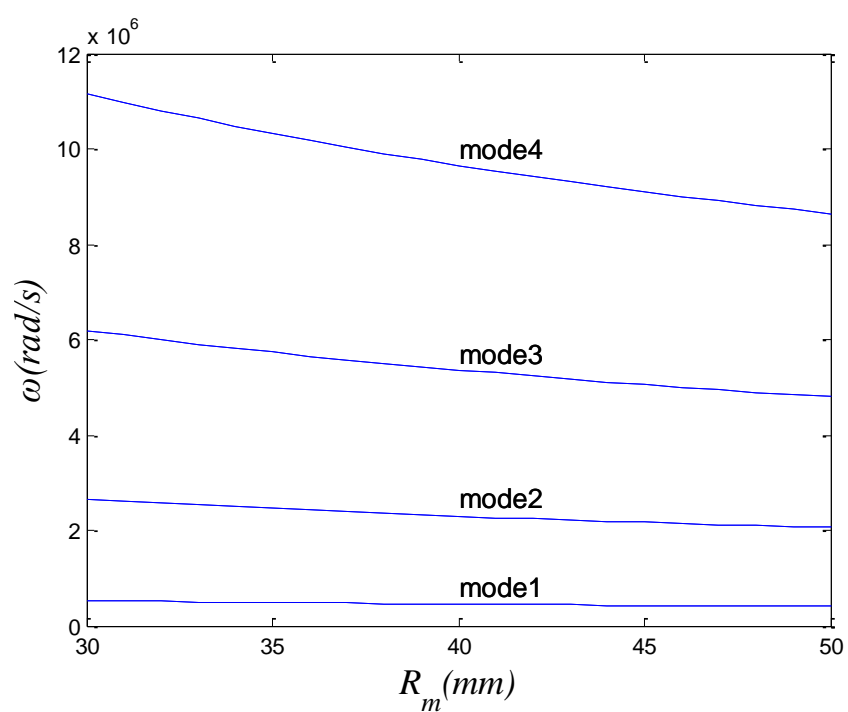

(e) $w_{0}$ changes

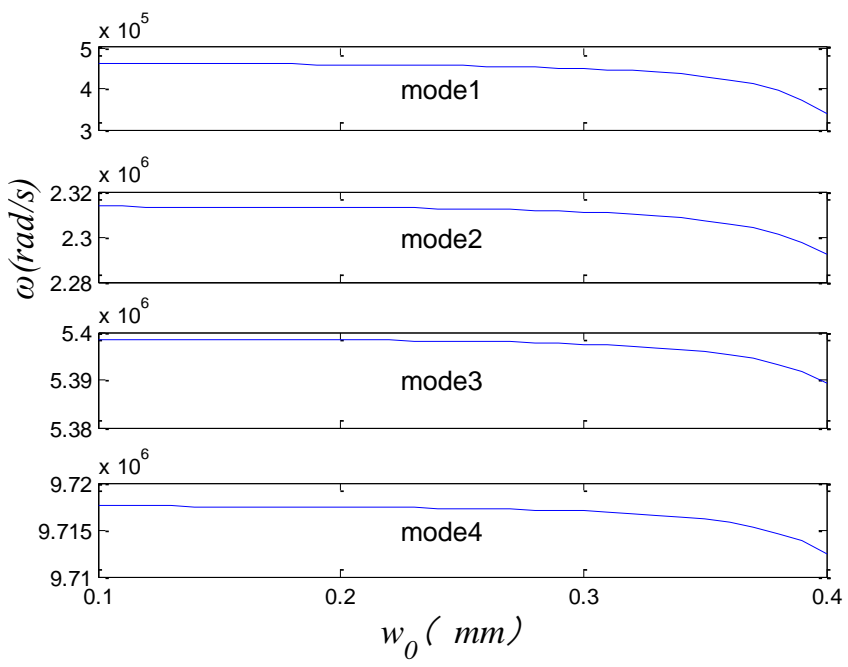

(f) $p$ changes

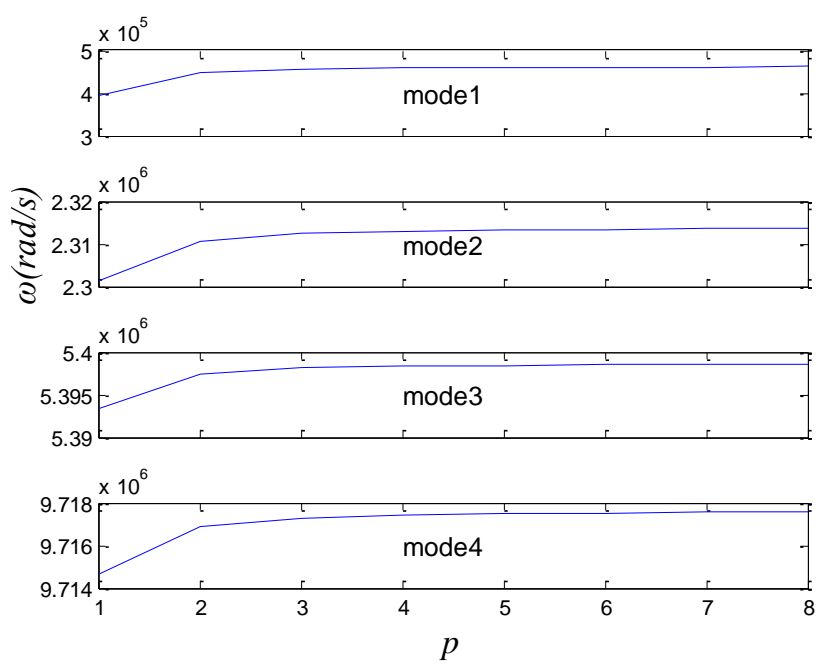

Fig. (4). Changes of the natural frequencies along with the main parameters. 
(a) mode 1

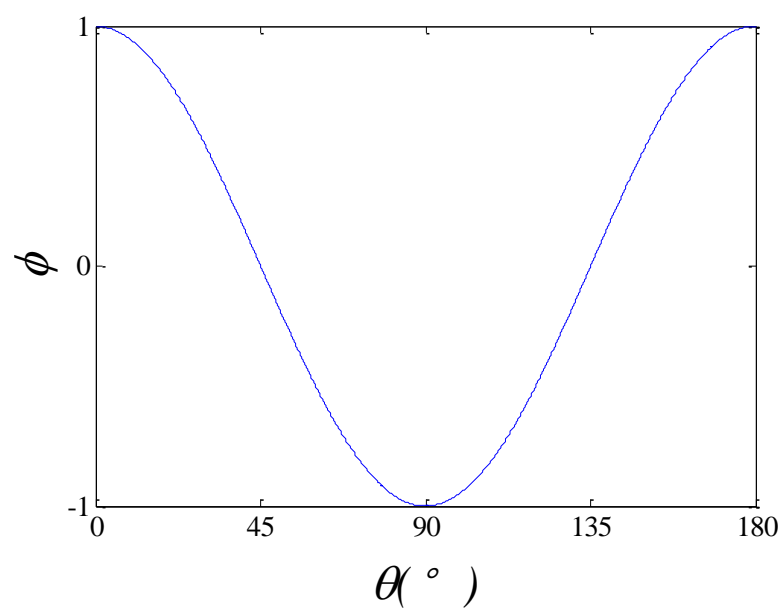

(b) mode 2

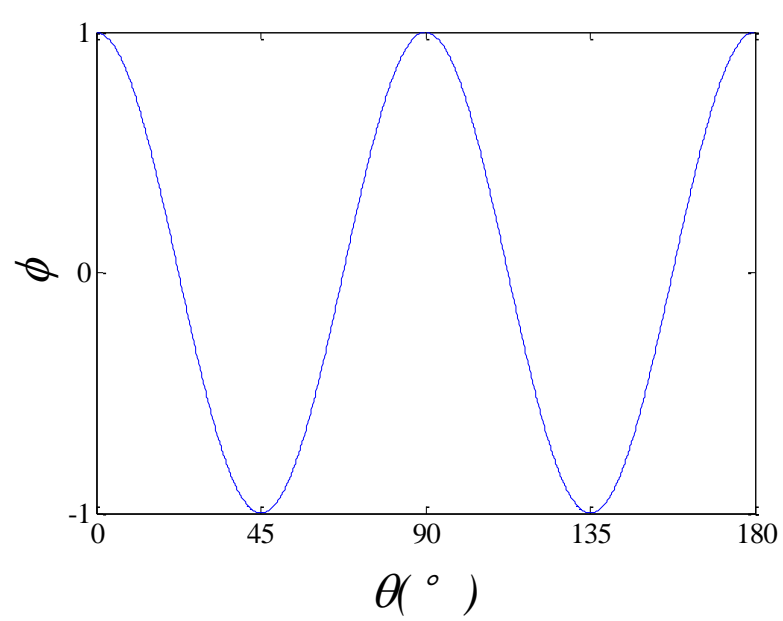

(c) mode 3

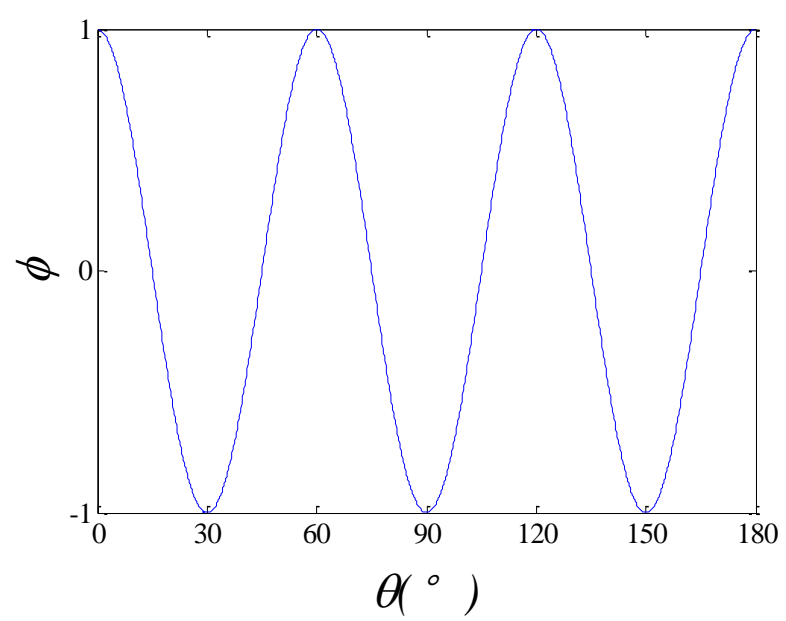

(d) mode 4

(Fig. 5) contd.....

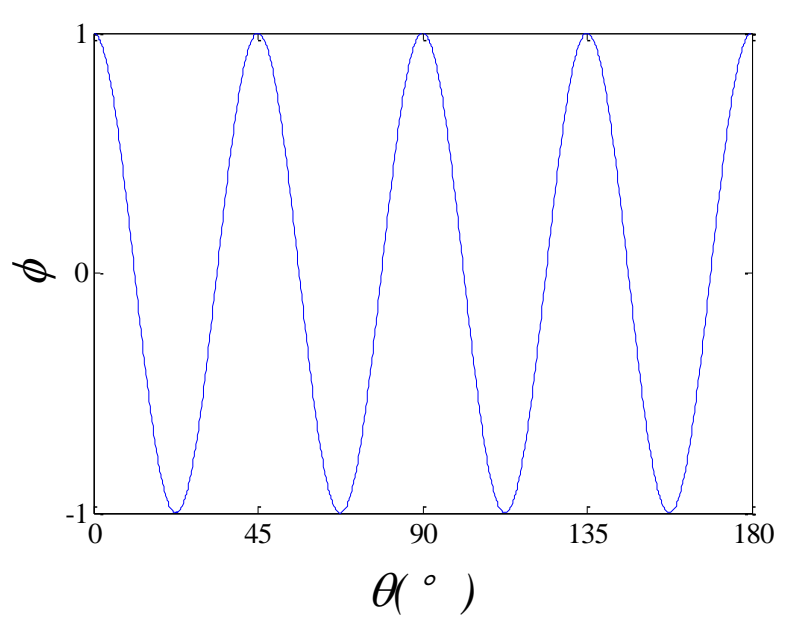

Fig. (5). Vibration modes of the flexible ring.

(5) As the average static displacement $w_{0}$ of the flexible ring increases, the natural frequency of the system decreases gradually. It is because a large static displacement $w_{0}$ can cause increase of the electromagnetic force for a given coil current. For the low order modes and a large static displacement $w_{0}$, the effects are more obvious.

(6) As the coil pole pair number grows, the natural frequency of the system increases gradually. For the low order modes and a small coil pole pair number, the natural frequency of the system increases more obviously with the increasing the coil pole pair number.

In a word, the natural frequency of the drive system is affected by mechanical and electrical parameters. Among them, the coil current $I$, the average static displacement $w_{0}$ of the flexible ring, and the clearance $\delta_{0}$ between the flexible ring and coils have the more obvious influence on the natural frequencies.

From Fig. (5), the following observations are worth noting:

(1) In mode 1, the maximum dynamic displacements occur at positions $=0^{\circ},=90^{\circ}$ and $=180^{\circ}$, and periodic time of the mode function equal . Among them, the dynamic displacements at positions $=0^{\circ}$ and $=180^{\circ}$ are in the same phase. They are in opposite phase with one at $=90^{\circ}$.

In mode 2, the maximum dynamic displacements occur at positions $=0^{\circ}, 45^{\circ}, 90^{\circ}, 135^{\circ}, 180^{\circ}$, and periodic time of the mode function equal $/ 2$. The dynamic displacements at positions $=0^{\circ}, \quad=90^{\circ}$ 
and $=180^{\circ}$ are in the same phase. They are in opposite phase with ones at $=0^{0}, 45^{0}$ and $135^{\circ}$.

In mode 3, the peak dynamic displacements occur still at positions $\theta=0^{\circ}, 30^{\circ}, 60^{\circ}, 90^{\circ}, 120^{\circ}, 150^{\circ}, 180^{\circ}$, and periodic time of the mode function equals $/ 3$. In mode 4 , five positive peak dynamic displacements and four negative peak dynamic displacements occur. The periodic time of the mode function equal $/ 4$.

Table 2. The first four natural frequencies ( $\mathrm{rad} / \mathrm{s})$.

\begin{tabular}{|c|c|c|c|}
\hline $\mathbf{1}$ & $\mathbf{2}$ & $\mathbf{3}$ & $\mathbf{4}$ \\
\hline \hline 458738 & 2312876 & 5398324 & 9717427 \\
\hline
\end{tabular}

\section{CONCLUSION}

In this paper, for an electromagnetic harmonic movable tooth drive system proposed, an electromechanical coupled dynamics equation of the flexible ring subjected to electromagnetic force is presented. Using the equation, the natural frequencies and vibration modes of the flexible ring are investigated. The results show that the natural frequency of the electromagnetic harmonic movable tooth drive system is affected by mechanical and electrical parameters among which, the coil current, the average static displacement of the flexible ring, and the air gap have more obvious influence on the natural frequencies.

\section{CONFLICT OF INTEREST}

The authors confirm that this article content has no conflict of interest.

\section{ACKNOWLEDGEMENTS}

This project is supported by National Natural Science Foundation of China (No. 51275441).

\section{REFERENCES}

[1] D. F. Herdeg, "Electromagnet harmonic drive low inertia serve", AD442879, Dec 1963.

[2] W. Trimmer, and R. Jebens, "Harmonic electrostatic motors", Sensors Actuators, vol. 20, pp. 17-24, 1989.

[3] O. Barth, "Harmonic piezodrive-miniaturized servo motor", Mechatronics, vol. 10, pp. 545-554, 2000.

[4] O. Kayabasi, and F. Erzincanli, "Shape optimization of tooth profile of a flexspline for a harmonic drive by finite element modeling", Mat. Design, vol. 28, pp. 441-447, 2007.

[5] C. F. Janes, "Electro-magnetic actuation", U.S. Patent 3200668, 1965.

[6] Z. Shang, and Z. You, "Control system for magnetic type harmonic gear drive", Manuf. Technol. Mach. Tool, vol. 3, pp. 7-10, 1997.

[7] J. Rens, K. Atallah, S. D. Calverley, and D. Howe, "A novel magnetic harmonic gear", IEEE Trans. Indust. Appl., vol. 46, pp. 206-212, 2010.

[8] Y. Ren, L. Xu, and Y. Wu, "Study on principle of electromechanical integrated electromagnetic harmonic oscillating teeth transmission", Int. J. Adv. Comput. Technol., pp. 183-192, 2012.

[9] R. Liu, C. Li, and Y. Zhang, "Analytical model for air-gap magnetic field calculation in an eccentric magnetic harmonic gear", In: Proce. Chin. Soc. Elect. Eng., vol. 33, pp. 126-133, 2013.

[10] U. Yutaka, and S. Yasushi, "Cyclic disturbance suppression control for harmonic magnetic gear", IEEE Trans. Indust. Appl., vol. 134, pp. 284-292, 2014.

[11] R. Dhaouadi, and F. H. Ghorbel, "Modelling and analysis of nonlinear stiffness, hysteresis and friction in harmonic drive gears", Int. J. Model. Simulat., vol. 28, no. 3, pp. 329-336, 2008.

[12] H. Dong, Z. Zhu, and W. Zhou, "Dynamic simulation of harmonic gear drives considering tooth profiles parameters optimization", $J$. Comput., vol. 7, no. 6, pp. 1429-1436, 2012.

[13] H. Zhu, P. Ning and M. Zou, "A gear pump based on harmonic gear drive”, J. Mech. Eng. Sci., vol. 227, no. 12, pp. 2844-2848, 2013. 